\title{
A Digital Hygrometer Using a Polyimide Film Relative Humidity Sensor
}

\author{
Hideo Shibata, Masahiro Ito, Masahiro Asakursa, and Kenzo Watanabe, Fellow, IEEE
}

\begin{abstract}
A digital hygrometer using a relative humidity sensor is developed. The sensor consists of a polyimide film spin-coated onto an Si substrate. The sensor model for signal processing, which describes the sensor capactiance in terms of relative humidity and temperature in closed form, is derived first. The interface appropriate for the sensor model, based on a relaxation oscillator, is designed next. Finally, a prototype hygrometer built using a one-chip microprocessor and its performance are described to demonstrate the validity of the sensor model and interface.
\end{abstract}

\section{INTRODUCTION}

$\mathbf{H}$ UMIDITY control, besides being used in industrial processes for the manufacture of products such as textiles, food, lumber, paper, and semiconductors, has recently been introduced into our daily life to make living and working environments comfortable. Toward this application, low-cost, mass-producible humidity sensors have been extensively developed. They are classified into the conductive type using ceramics or aluminum oxides and the capacitive type using polymer films. The conductance of the conductivetype humidity sensor increases exponentially with adsorption of water vapor in the air [1]-[3]. Their sensitivity is high enough, but is degraded seriously by surface contaminations due to exhaust and dust. Their large temperature dependence is another problem.

The capacitive-type sensor makes use of the increase of apparent dielectric permittivity due to moisture absorption. It therefore tolerates pollution, and is suited for environment assessment. Hygroscopic polymer films such as lithium-fluoride [4], cellulose acetate [5], and cellulose acetate butyrate [6] have been used in the early stages of the development. Compared with these hydrophilic films, the hydrophobic film using polyimide is inferior in sensitivity but is superior in long-term stability. As with all other sensors, the long-term stability rather than the sensitivity is emphasized in their field applications. Recent work has, therefore, concentrated on the polyimide-based humidity sensors [7]-[11].

A digital hygrometer described in this paper also uses a polyimide capacitor as the sensing element. Its capacitance changes nonlinearly with relative humidity $(\mathrm{RH})$ and temper-

Manuscript received April 24, 1995; revised January 3, 1996.

H. Shibata, M. Ito, and M. Asakura are with the Development Laboratory, Kurabe Industrial Corporation, 4830 Takatsuka, Hamamatsu, 432 Japan.

$\mathrm{K}$. Watanabe is with the Research Institute of Electronics, Shizuoka University, 3-5-1 Johoku, Hamamatsu, 432 Japan.

Publisher Item Identifier S 0018-9456(96)03494-8.

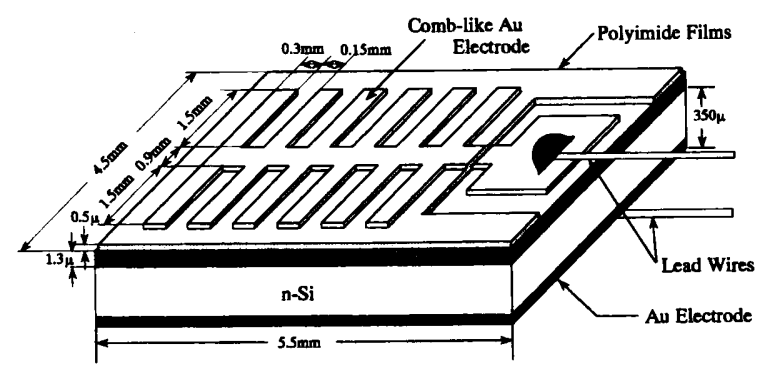

Fig. 1. Structure of the relative humidity sensor.

ature. The temperature dependence is small compared to the dielectric sensitivity, i.e., the capacitance change due to $\mathrm{RH}$, but cannot be neglected for accurate measurements. Therefore, the sensor model which takes the temperature dependence into account is first established for signal processing. The capacitance change should be detected in a digital form for linearization. The simplest circuit to perform such a function is a relaxation oscillator. A high sensitivity cannot be expected from its conventional form, however, because the capacitance change of the sensor due to $\mathrm{RH}$ is small compared to its offset capacitance when $\mathrm{RH}=0$, which is referred to hereafter as the dry capacitance. To encode only the capacitance change into a digital form, a relaxation oscillator which cancels the dry capacitance is developed. Finally, a prototype hygrometer based on these techniques will be described to demonstrate their validity.

\section{SEnSor AND Its Signal Processing Model}

Fig. 1 shows the structure of the RH sensor. Its fabrication process is as follows. First, Au is evaporated onto one surface of a $0.001 \Omega \cdot \mathrm{cm}, \mathrm{n}-\mathrm{Si}$ substrate $350 \mu \mathrm{m}$ thick, and is sintered at $650^{\circ} \mathrm{C}$ for $10 \mathrm{~min}$ to make the contact ohmic. After removing an $\mathrm{SiO}_{2}$ layer on the other surface by chemical etching, the polyimide is spin-coated onto the etched surface at $3000 \mathrm{r} / \mathrm{min}$ for $30 \mathrm{~s}$. The polyimide film is prebaked at $200^{\circ} \mathrm{C}$ for $1 \mathrm{~h}$ and then cured at $350^{\circ} \mathrm{C}$ for $1 \mathrm{~h}$. The hygroscopic film thus fabricated is typically $1.3 \mu \mathrm{m}$ thick. Then, a comb-like Au electrode is sputtered onto the film. The electrode is so thin, typically $500 \AA$, and porous that it is transparent to moisture. The comb-like configuration is adopted for quick absorption and desorption of water vapor. A thin, typically $50 \mu \mathrm{m}$ thick, polyimide film is again spin-coated all over the hygroscopic film to protect it from mechanical damage in the subsequent bonding and packaging process. This wafer is then scribed into 


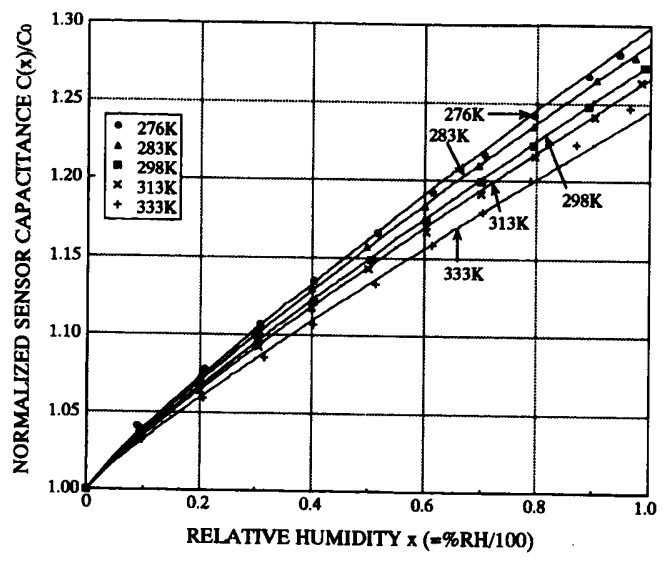

Fig. 2. Capacitance $C(x)$ of the RH sensor normalized by its dry capacitance $C_{0}$ and its functional fitting (solid lines).

TABLE I

Comparison of the Fractional CapactTance Changes of RH Sensors Using Polyimide Films

\begin{tabular}{|c|c|c|c|c|}
\hline Sensor & Thickness & $\left.C_{(x)}\right) C_{0}$ & Condition & Cure Proces: \\
\hline A $[7]^{2}$ & $2.6 \mu \mathrm{m}$ & 1.27 & 97\% RH, RT & $30 \min @ 90^{\circ} \mathrm{C}, 1 \mathrm{~h} @ 350^{\circ} \mathrm{C}$ \\
\hline$B[8]$ & $1.38 \mu \mathrm{m}$ & 1.27 & $100 \% \mathrm{RH}, 30^{\circ} \mathrm{C}$ & $30 \min @ 150^{\circ} \mathrm{C}, 11 @ 250^{\circ} \mathrm{C}, 20 \min @ 425^{\circ} \mathrm{C}$ \\
\hline c [9] & $1.5 \mu \mathrm{m}$ & 1.26 & $90 \% \mathrm{RH}, 35^{\circ} \mathrm{C}$ & $1 \mathrm{~h} @ 200^{\circ} \mathrm{C}, 1 \mathrm{~h} @ 350^{\circ} \mathrm{C}$ \\
\hline$D[100]$ & $5.5 \mu \mathrm{m}$ & 1.31 & 95\%RH, RT ${ }^{3}$ & $15 \min @ 90^{\circ} \mathrm{C}, 45 \min @ 3350^{\circ} \mathrm{C}$ \\
\hline$E^{2)}$ & $1.3 \mu \mathrm{m}$ & 1.275 & $100 \%$ RH, $25^{\circ} \mathrm{C}$ & $1 \mathrm{~h} @ 200^{\circ} \mathrm{C}, 1 \mathrm{~h} @ 350^{\circ} \mathrm{C}$ \\
\hline
\end{tabular}

1) The number in brackets is the reference number.

2) Present work, 3) Room Temperature

chips measuring $4.5 \mathrm{~mm} \times 5.5 \mathrm{~mm}$. Each chip is mounted in a perforated metal package.

The capacitance of the $\mathrm{RH}$ sensor measured at $20 \mathrm{kHz}$ is plotted in Fig. 2 as a function of $\mathrm{RH}$ and temperature. The abscissa is $\mathrm{RH}$ measured using a commercial dew-point indicator with $\pm 0.1{ }^{\circ} \mathrm{C}$ accuracy, and the ordinate is the sensor capacitance $C(x)$ normalized by its dry capacitance $C_{0}$ when $\mathrm{RH}=0 . C_{0}$ is typically $300 \mathrm{pF}$. The temperature dependence of $C_{0}$ is so small, $-7 \times 10^{-3} \%{ }^{\circ} \mathrm{C}$, that it is neglected in the following discussions. Table I compares the fractional capacitance change with those reported elsewhere. This comparison shows that the fractional capacitance changes of the polyimide-based RH sensors are almost independent of their fabrication process and film thickness. The response time for the step change between 50 and $90 \% \mathrm{RH}$ is shorter than $15 \mathrm{~s}$. These facts indicate that the capacitance change of the sensor can be explained by the increase in the apparent dielectric constant $\epsilon_{s}$ of hygroscopic film due to physical sorption of water vapor. Absorbed water can be regarded reasonably as being distributed uniformly throughout the film because the fractional capacitance change is independent of the film thickness. Therefore, the sensor capacitance $C(x)$ is given by

$$
\frac{C(x)}{C_{0}}=\frac{L_{p}}{\epsilon_{p}} \int_{0}^{L_{p}} \frac{d l}{\epsilon_{s}}=\frac{\epsilon_{s}}{\epsilon_{p}}
$$

where $\epsilon_{p}$ is the dielectric constant of the polyimide $\left(\epsilon_{p}=2.7\right)$ and $L_{p}$ is the thickness of the hygroscopic film.

The apparent dielectric constant $\epsilon_{s}$ of the hygroscopic film is given by Looyenga's empirical equation [8], [12]:

$$
\epsilon_{s}=\left\{\gamma\left(\epsilon_{w}^{1 / 3}-\epsilon_{p}^{1 / 3}\right)+\epsilon_{p}^{1 / 3}\right\}^{3}
$$

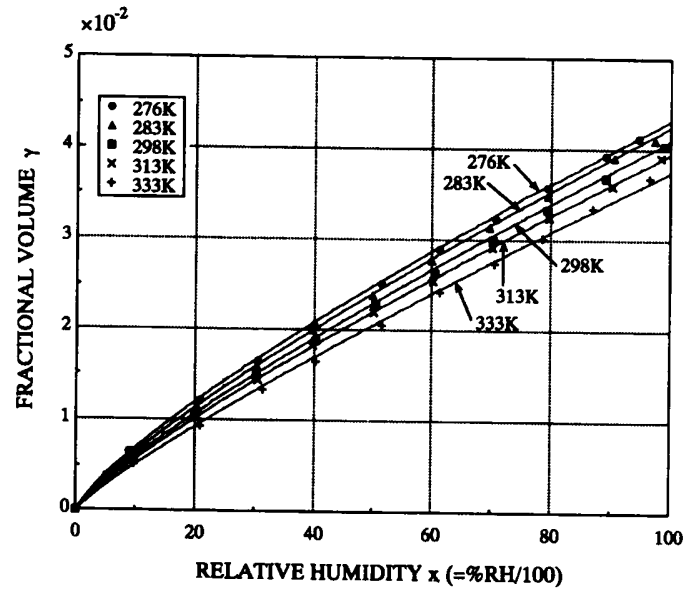

Fig. 3. Fractional volume $\gamma$ of water in a polyimide film.

where $\gamma$ is the fractional volume of water in the film and $\epsilon_{w}$ is the dielectric constant of water given by [13]

$$
\begin{aligned}
\epsilon_{w}=78.54\left\{1-4.6 \times 10^{-4}(\right. & T-298) \\
+ & \left.8.8 \times 10^{-6}(T-298)^{2}\right\}
\end{aligned}
$$

where $T$ is temperature in kelvin. The fractional volume $\gamma$ can be obtained from the measured capacitance $C(x)$ using (1) and (2). The results are plotted in Fig. 3. The plots can be approximated by the following function of $\mathrm{RH}$ and temperature:

$$
\gamma=\gamma_{m} \phi(T) x^{\psi(T)}
$$

where

$$
\begin{gathered}
x=\% \mathrm{RH} / 100 \\
\phi(T)=1-\alpha_{0}\left(T-T_{0}\right) \\
\psi(T)=\psi_{0} \frac{1-\alpha_{1}\left(T-T_{0}\right)+\alpha_{2}\left(T-T_{2}\right)^{2}}{1+\beta_{1} \exp \left\{\beta_{2}\left(T-T_{0}\right)\right\}}
\end{gathered}
$$

and $\gamma_{m}$ is the maximum fractional volume at $T_{0}$. Physically, $\phi(T)$ represents the temperature dependence of the adsorption coefficient, while $\psi(T)$ reflects the temperature dependence of $\epsilon_{w}$ and the catalytic effect. This physical interpretation indicates that a Fickian model is applicable to the diffusion process. The solid lines in Fig. 3 show $\gamma$ calculated using (4) with the following parameter values: $\gamma_{m}=4.04 \times 10^{-2}, T_{0}=$ $298 \mathrm{~K}, \alpha_{0}=2.43 \times 10^{-3}, \psi_{0}=0.836, \alpha_{1}=2.22 \times 10^{-4}$, $\alpha_{2}=2.34 \times 10^{-5}, \beta_{1}=4.9 \times 10^{-3}$, and $\beta_{2}=-0.12$. Each line fits the experimentally obtained data quite well. The value of $\gamma_{m}$ is also in good agreement with the water absorption coefficient of the polyimide. Substituting (4) into (2), one can obtain the fractional capacitance of the RH sensor as a function of RH and temperature. The resultant expression is, however, too complicated to be processed by an interface circuit or even by software.

Fig. 2 indicates that the fractional capacitance versus $\mathrm{RH}$ characteristics at $T$ can be obtained by scaling the reference characteristics at $T_{0}=298 \mathrm{~K}$. From this observation, a much simpler expression can be derived as follows: The reference 
characteristics can be described in terms of the catalytic function

$$
\frac{C\left(x, T_{0}\right)}{C_{0}}=\frac{\epsilon_{0}+\epsilon_{d} x}{1+\left(\epsilon_{0}-1\right) \exp \left(-\frac{x}{\lambda}\right)}
$$

where $\lambda$ is the catalytic factor and $\epsilon_{d}$ is the dielectric sensitivity at $T_{0}$ given by

$$
\epsilon_{d}=\frac{\epsilon_{s}\left(T_{0}\right)}{\epsilon_{p}}-1=\left[\gamma_{m}\left\{\left(\frac{\epsilon_{w}\left(T_{0}\right)}{\epsilon_{p}}\right)^{1 / 3}-1\right\}+1\right]^{3}-1 .
$$

Equation (8) can be well approximated by the nonlinear function $1+x^{n}$, where $n$ is a nonlinear index smaller than 1. Including the temperature scaling factor $\chi(T)$ into $x$, one can derive the signal processing model of the sensor:

$$
\frac{C(x, T)}{C_{0}}=1+\epsilon_{d}\left(\frac{x}{\chi(T)}\right)^{n}
$$

where $\chi(T)$ is given by

$$
\chi(T)=\frac{1+\theta_{1}\left(T-T_{0}\right)+\theta_{2}\left(T-T_{0}\right)^{2}}{1+\rho_{1} \exp \left(-\frac{T-T_{0}}{\rho_{2}}\right)} .
$$

It should be noted that $\chi(T)$ includes the temperature dependence of the permittivity and the catalytic effect. The dielectric sensitivity $\epsilon_{d}$ and the nonlinear index $n$ can be obtained from the sensor capacitances measured at two points of $\mathrm{RH}$ at the reference temperature, while the other coefficients, $\theta_{1}, \theta_{2}$, $\rho_{1}$, and $\rho_{2}$, from the sensor capacitances measured at some, say $50 \%, \mathrm{RH}$ at different temperatures $T_{i}(i=1,2,3,4)$. Their values thus obtained are: $\epsilon_{d}=0.275, n=0.884$, $\theta_{1}=1.67 \times 10^{-3}, \theta_{2}=6.65 \times 10^{-5}, \rho_{1}=10^{-2}$, and $\rho_{2}=10 . T_{0}=298 \mathrm{~K}$ is assumed. Fig. 4 shows the temperature scaling factor $\chi(T)$ calculated by (11) using these values. Dots indicate $\chi(T)$ values obtained from the measured sensor capacitances. The fractional capacitances calculated by (10) are shown in Fig. 2 by solid lines. Each line fits the measured values quite well, except at the high temperature and high $\mathrm{RH}$ region. This comparison confirms, therefore, that (10) gives an exact model of the polyimide-based RH sensor for its signal processing. The advantage of this model is that its parameters can be uniquely determined by six-point measurements in the specified $\mathrm{RH}$ and temperature range. The interface based on this model will be described next.

\section{INTERFACE}

An oscillation period of a conventional relaxation oscillator consisting of an integrator followed by a comparator is proportional to the integration time constant $\tau_{c}=C R$ [14]. Therefore, using the capacitor under measurement as the integration capacitor is the easiest way of detecting its capacitance in a digital form [15], [16]. In the present case, however, the physical quantity to be detected is $\mathrm{RH}$, which is related to a capacitance measurement by (10):

$$
\% \mathrm{RH}=\chi(T)\left\{\frac{1}{\epsilon_{d}} \frac{C(x)-C_{0}}{C_{0}}\right\}^{1 / n} \times 100 .
$$

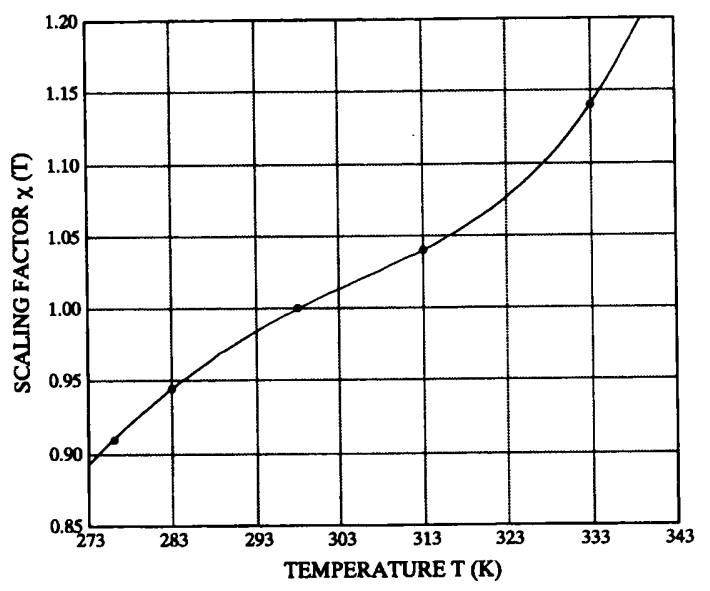

Fig. 4. Experimentally obtained values (dots) of the temperature scaling factor $\chi(T)$ and their functional fitting (solid line).

This relation requires the integration time constant to be proportional to the capacitance difference. This requirement can be expressed in a transfer function form of an integrator as follows:

$$
H(s)=\frac{V_{0}(s)}{V_{i}(s)}=-\frac{1}{s R\left(C(x)-C_{0}\right)} .
$$

Arranging (13) into the state-variable form

$$
V_{0}(s)=-\frac{1}{s R C(x)}\left(V_{i}(s)-s R C_{0} V_{0}(s)\right),
$$

one finds that the required transfer function can be realized by an integrator and a differentiator.

The interface based on the above synthesis is shown in Fig. 5(a). Op-amp $A_{2}$ is added to the relaxation oscillator consisting of op-amp $A_{1}$ and comparator $A_{3}$ to perform the derivative operation involved in (14). The RH sensor is denoted by $C(x)$. Fig. 5(b) shows the voltage waveform $v_{A}$ at the noninverting input terminal (A) of comparator $A_{3}$, assuming the bias voltage $V_{\text {bias }}=0$. Referring to Fig. 5(b), one can derive the following expression for the oscillation period $\tau$ :

$$
\tau=\tau_{1}+\tau_{2}=\kappa\left(2+\zeta+\frac{1}{\zeta}\right) R_{1}\left\{C(x)-\frac{R_{2}}{R_{3}} C_{1}\right\}
$$

where

$$
\begin{gathered}
\kappa=\frac{R_{4}}{R_{5}} \\
\zeta=\frac{V_{n}}{V_{p}}
\end{gathered}
$$

and $V_{p}$ and $V_{n}$ are the output voltage levels of $A_{3}$ when $v_{A} \geq 0$ and $v_{A}<0$, respectively. In deriving (15), the ideal op-amps and comparator are assumed. When op-amps $A_{1}$ and $A_{2}$ have offset voltages $V_{o s 1}$ and $V_{o s 2}$, respectively, and the comparator has response delay $\tau_{d}$, the period is modified, to first order, to

$$
\tau^{\prime}=\tau+\Delta \tau
$$

where

$$
\Delta \tau=\tau_{d}\left(2+\zeta+\zeta^{-1}\right)-2\left(\frac{V_{o s 1}}{V_{p}}\right) C_{1} R_{1} R_{2} / R_{3} .
$$




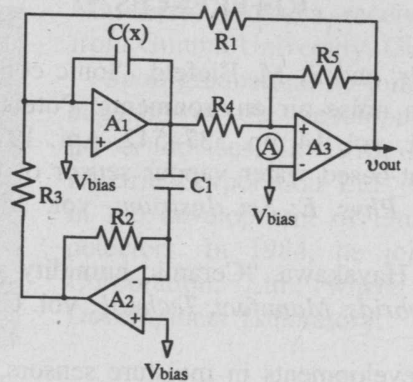

(a)

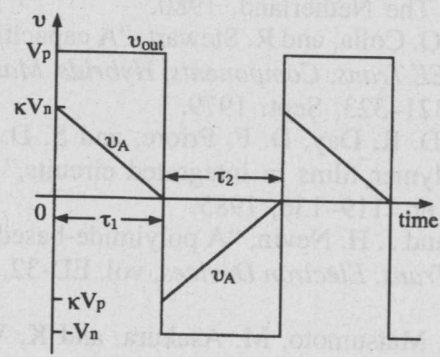

(b)

Fig. 5. (a) Circuit diagram and (b) voltage waveforms of the interface.

The error term $\Delta \tau$ does not include $V_{o s 2}$ because its firstorder effects on $\tau_{1}$ and $\tau_{2}$ cancel each other. This also holds true for the offset voltage of $A_{3}$. It should also be noted that $\Delta \tau$ is independent of $C(x)$ and affects only the offset in $\tau$. Substituting (15) into (18), one notices that if $R_{2}$ is adjusted such that

$$
\frac{R_{2}}{R_{3}} C_{1}\left(1+\frac{2}{2+\zeta+\zeta^{-1}} \frac{V_{o s 1}}{\kappa V_{p}}\right)-\frac{\tau_{d}}{\kappa R_{1}}=C_{0}
$$

hold, then the interface provides the period exactly proportional to the capacitance change $\Delta C(x)=C(x)-C_{0}$ of the RH sensor. The proportional constant, i.e., the conversion sensitivity, can be adjusted by means of $R_{1}$ without affecting the offset adjustment if $\tau_{d}$ is small.

\section{PROTOTYPE HYgROMETER}

A digital hygrometer using a polyimide-film $\mathrm{RH}$ sensor was built based on the sensor model and interface described in the previous sections. Measurements from 10 to $90 \% \mathrm{RH}$ with $\pm 1 \%$ accuracy are specified over the temperature range from 10 to $40^{\circ} \mathrm{C}$.

Its block diagram and picture are shown in Fig. 6. It consists of the probe and signal processing unit connected by four wires, two for the power supply and the other two for signal transmission. The probe incorporates, besides the RH sensor and its interface, a thermistor and its interface for temperature detection. The thermistor interface takes the same circuit configuration as that shown in Fig. 5(a), but $C(x)$ is replaced by a fixed capacitor and the thermistor is connected in place of $R_{1}$. For linearization, two resistors, one connected in parallel and the other in series with the thermistor, are used.

An $8 \mathrm{~b}$, one-chip microcomputer is used for detection of the oscillation periods and subsequent signal processing. It accommodates two ROM tables: one referred to as the RH table, and the other as the temperature scaling (TS) table. The RH table stores $N^{1 / n}$ in the address $N$, while the TS table the

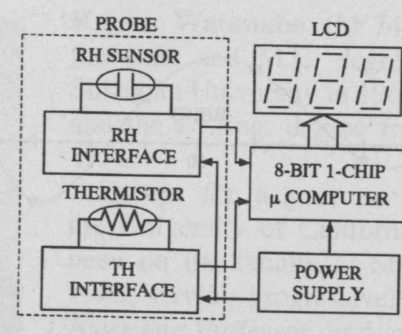

(a)

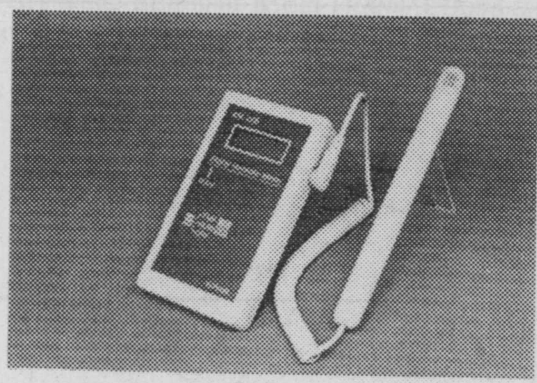

(b)

Fig. 6. (a) Block diagram of (b) prototype hygrometer.

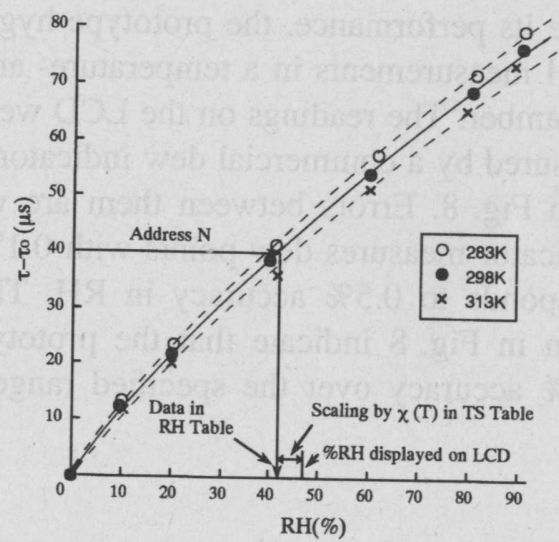

Fig. 7. Measured oscillation period of the interface oscillator and the table look-up procedure.

scaling factor $\chi(T)$ calculated using (11). Their addresses are generated by detecting the oscillation periods of the interface oscillators using a $500 \mathrm{kHz}$ clock signal. The period $\tau$ given by (15) becomes too short to be measured accurately in the low RH region when $R_{2}$ is adjusted to meet (20). To avoid this difficulty, $\tau_{0}=165 \mu$ s offset is introduced into the RH interface by offsetting $R_{2}$. This lowers the oscillation frequency and is also desirable for the stable operation of the op-amp-based relaxation oscillator. The measured period $\tau$ is plotted in Fig. 7 as a function of RH with temperature as a parameter. For better resolution in the low RH region, the microcomputer counts the clock pulse over 30 periods of $\tau$ to generate the address $N$. The address $N$ indicated in Fig. 7 excludes the offset $\tau_{0}$, and thus corresponds to $C(x)-C_{0}$. The content in the address $N$ is $N^{1 / n}$, which corresponds to \% RH at the reference temperature $T=298 \mathrm{~K}$, as given by (12). Scaling it by the scaling factor obtained from the TS table accessed by a similar procedure, the microcomputer displays $\% \mathrm{RH}$ on the LCD. The above procedure is also illustrated in Fig. 7. 


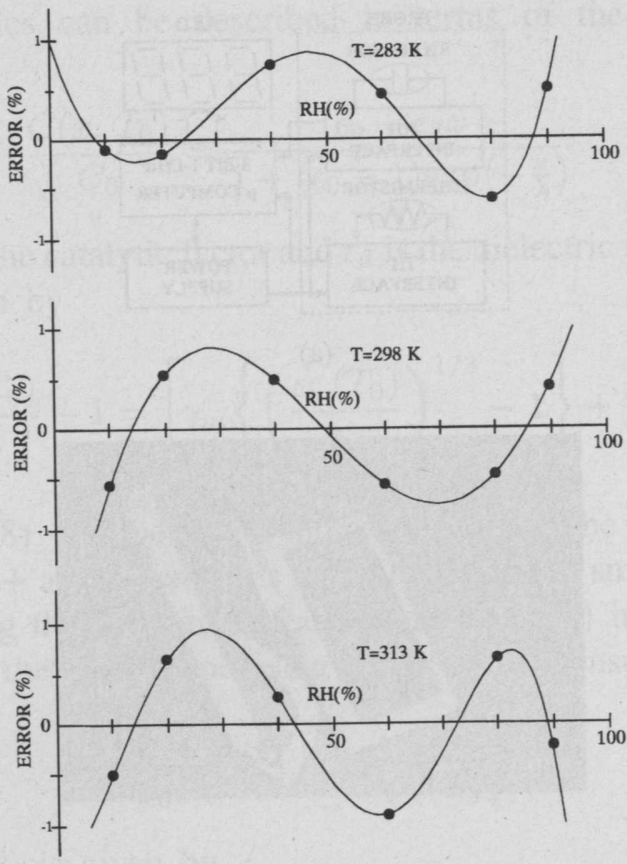

Fig. 8. Errors between RH measured by the prototype hygrometer and that by a commercial dew indicator.

To evaluate its performance, the prototype hygrometer was applied to RH measurements in a temperature- and humiditycontrolled chamber. The readings on the LCD were compared to those measured by a commercial dew indicator. The results are plotted in Fig. 8. Errors between them are within $\pm 1 \%$. The dew indicator measures dew points with $0.1^{\circ} \mathrm{C}$ accuracy, which corresponds to $0.5 \%$ accuracy in $\mathrm{RH}$. Therefore, the results shown in Fig. 8 indicate that the prototype hygrometer has $\pm 1 \%$ accuracy over the specified range of $\mathrm{RH}$ and temperature.

\section{CONCLUSIONS}

A signal processing model of an RH sensor using a polyimide film was derived based on the physical adsorption and absorption of water vapor. We believe this to be the first model which expresses the nonlinearity and the temperature dependence in a closed form. An interface appropriate for this model was then developed. A prototype hygrometer built based on these techniques has met the stringent requirement of $\pm 1 \%$ accuracy in RH measurement over a $10-90 \% \mathrm{RH}$ and $10-40^{\circ} \mathrm{C}$ range. This proves the validity of the sensor model and the interface. The component requirement for its implementation is minimal; it was built in practice using only six off-theshelf IC-chips including the $5 \mathrm{~V}$ power supply. The digital hygrometer developed in this work thus has the advantage of high performance/cost. This salient feature makes it applicable not only to humidity control of living environments, but also to industrial processes. Toward such applications, its performance evaluation in a wider temperature range is now underway.

\section{ACKNOWLEDGMENT}

The authors thank M. Nakayama for his efforts in preparing the manuscript.

\section{REFERENCES}

[1] R. T. Johnson, Jr. and R. M. Biefeld, "Ionic conductivity of $\mathrm{Li}_{5} \mathrm{AlO}_{4}$ and $\mathrm{Li}_{5} \mathrm{GaO}_{4}$ in noise air environments: Potential humidity sensor," Mater. Res. Bull., vol. 14, pp. 537-542, Apr. 1979.

[2] P. Wiedjik, "Flat-based water vapour sensor of the phosphorous pentoxide type," J. Phys. E: Sci. Instrum., vol. 13, pp. 993-994, Sept. 1980.

[3] T. Nitta and S. Hayakawa, "Ceramic humidity sensors," IEEE Trans. Components, Hybrids, Manufact. Technol., vol. CHMT-3, pp. 237-241, June 1980.

[4] L. C. Yang, "Developments in moisture sensors," Meas. Control, vol. 15, pp. 98-108, Feb. 1981.

[5] "Capacitive humidity sensor," Tech. Inform. 063, Philips Nederland B. V., Eindhoven, The Netherland, 1980.

[6] P. E. Thoma, J. O. Colla, and R. Stewart, "A capacitive humidity-sensing transducer," IEEE Trans. Components, Hybrids, Manufact. Technol., vol. CHMT-2, pp. 321-323, Sept. 1979.

[7] D. D. Denton, D. R. Day, D. F. Priore, and S. D. Senturia, "Moisture diffusion in polymer films in integrated circuits," J. Electron. Mater., vol. 14 , no. 2, pp. 119-136, 1985.

[8] D. J. Schubert and J. H. Nevin, "A polyimide-based capacitive humidity sensor," IEEE Trans. Electron Devices, vol. ED-32, pp. 1220-1223, July 1985.

[9] H. Shimizu, H. Matsumoto, M. Asakura, and K. Watanabe, "A digital hygrometer," IEEE Trans. Instrum. Meas., vol. 37, pp. 300-304, June 1988.

[10] D. D. Denton, C. N. Ho, and S-G. He, "A solid-state relative humidity measurement system," IEEE Trans. Instrum. Meas., vol. 39, pp. 508-511, June 1990.

[11] T. Kuroiwa, T. Hayashi, A. Ito, M. Matsuguchi, Y. Sadaoka, and Y. Sakai, "A thin film polyimide based capacitive type relative humidity sensor," Sensors and Actuators B, vol. 13-14, pp. 89-91, 1993.

[12] M. M. Dubinin and V. A. Astakhov, "Description of absorption equilibria of vapors on zeolites over wide ranges of temperature and pressure," Molecular Sieve Zeolites, vol. II, R. F. Gould, Ed., in Adv. in Chemistry Series, no. 102. New York: Amer. Chem. Soc., 1971.

[13] J. B. Hasted, Aqueous Dielectrics. London: Chapman and Hall, 1973, pp. $37-38$

[14] A. S. Sedra and K. C. Smith, Microelectronic Circuits. Philadelphia: Saunders College Publishing, 1991, ch. 12.

[15] B. K. Ward, "Digital hygrometer with capacitive sensor," J. Phys. E: Sci. Instrum., vol. 16, pp. 731-733, 1983.

[16] F. Krummenacher, "A high-resolution capacitance-to-frequency converter," IEEE J. Solid-State Circuits, vol. SC-20, pp. 666-670, June 1985.

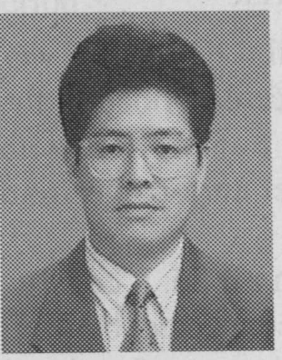

Hideo Shibata received the B.Eng. degree from the Tokyo University of Agriculture and Technology, Tokyo, Japan, in 1987.

$\mathrm{He}$ joined Kurabe Industrial Company, Ltd., Hamamatsu, Japan, in 1987, and is now leading the circuit design group in the Development Laboratory.

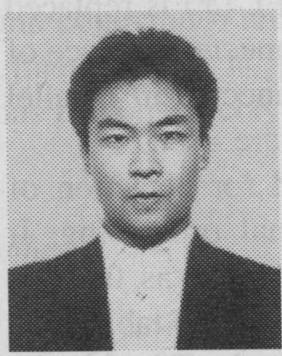

Masahiro Ito graduated from the College of Engineering, Shizuoka University, Hamamatsu, Japan, in 1989.

He joined Kurabe Industrial Company, Ltd., Hamamatsu, Japan, in 1989 , and is now engaged in the development of measurement and control circuits in the Development Laboratory. 


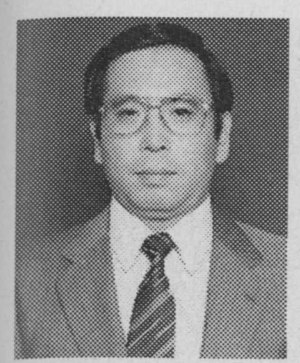

Masahiro Asakura received the B.Eng. degree from Gunma University, Gunma, Japan, in 1967.

Upon graduation, he joined NEC, where he was engaged in the development of semiconductor power devices. In 1975, he moved to Shibaura Electric Corporation Ltd., where he was engaged in the development of temperature and humidity detectors. In 1984, he joined Kurabe Industrial Corporation, Ltd., where he is managing the Development Laboratory.

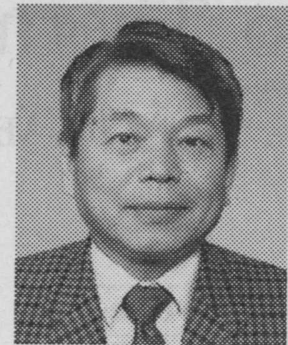

Kenzo Watanabe (M'74-SM'86-F'93) received the B.E. and M.E. degrees in engineering from Shizuoka University in 1962 and 1966, respectively, and the Dr.Eng. degree from Kyoto University in 1976.

Except for a year as a Visiting Professor at the University of California, Los Angeles, he has been on the faculty at Shizuoka University since 1962, serving progressively as Research Assistant, Associate Professor, and currently Professor in the Research Institute of Electronics.

Dr. Watanabe is involved in IEEE activities as an AdCom member of the IM Society, an Associate Editor of IEEE TRANSACTIONS ON INSTRUMENTATION AND MEASUREMENT, and a member of the IMTC Board of Directors. 